\title{
Climate change is catchy - but when will it really hurt?
}

\author{
N A Sweijd, ${ }^{1} \mathrm{PhD} ; \mathbf{C}$ Y Wright, ${ }^{2} \mathrm{PhD}$; A Westwood, ${ }^{3} \mathrm{MB} \mathrm{ChB}$, FCP (SA), MD; M Rouault, ${ }^{4} \mathrm{PhD} ; \mathbf{~ W}$ A Landman, ${ }^{5} \mathrm{PhD}$; \\ M L MacKenzie, ${ }^{6} \mathrm{PhD} ;$ J J C Nuttall, ${ }^{7} \mathrm{MB}$ ChB, FCPaed (SA), DTM\&H; H Mahomed, ${ }^{8} \mathrm{MB}$ ChB; T Cousins, ${ }^{9} \mathrm{PhD} ; \mathbf{K}$ Winter, ${ }^{10} \mathrm{PhD}$; \\ F Berhoozi, ${ }^{11}$ MA, MPH; B Kalule, ${ }^{12}$ BVSc, MMed; P Kruger, ${ }^{13}$ Dip Pub Health; T Govender, ${ }^{8} \mathrm{PhD} ; \mathbf{N}$ Minakawa, ${ }^{14} \mathrm{PhD}$ \\ ${ }^{1}$ Applied Centre for Climate and Earth Systems Science, Council for Scientific and Industrial Research, Cape Town, South Africa \\ ${ }^{2}$ Environment and Health Research Unit, South African Medical Research Council and Department of Geography, Geoinformatics and \\ Meteorology, Faculty of Science, University of Pretoria, South Africa \\ ${ }^{3}$ Department of Paediatrics, Faculty of Health Sciences, University of Cape Town, South Africa \\ ${ }^{4}$ Department of Oceanography, Faculty of Science, University of Cape Town, South Africa \\ ${ }^{5}$ Natural Resources and the Environment, Council for Scientific and Industrial Research, Pretoria, South Africa \\ ${ }^{6}$ Centre for Research into Ecological \& Environmental Modelling, School of Mathematics \& Statistics, St Andrews University, Scotland, UK \\ ${ }^{7}$ Infectious Diseases Unit, Department of Paediatrics and Child Health, Red Cross War Memorial Children's Hospital and Faculty of Health \\ Sciences, University of Cape Town, South Africa \\ ${ }^{8}$ Metro District Health Services, Western Cape Government: Health and Division of Community Health, Faculty of Medicine and Health \\ Sciences, Stellenbosch University, Cape Town, South Africa \\ ${ }^{9}$ Department of Sociology and Social Anthropology, Faculty of Arts and Social Sciences, Stellenbosch University, Stellenbosch, South Africa \\ ${ }^{10}$ Department of Environmental \& Geographical Science, Faculty of Science, University of Cape Town, South Africa \\ ${ }^{11}$ School of Child and Adolescent Health, Faculty of Health Sciences, University of Cape Town, South Africa \\ ${ }^{12}$ Department of Pathology, Division of Medical Microbiology, Faculty of Health Sciences, University of Cape Town, South Africa \\ ${ }^{13}$ Malaria Institute, Department of Health Limpopo, Tzaneen, South Africa \\ ${ }^{14}$ Department of Vector Ecology and Environment, Institute of Tropical Medicine, Nagasaki University, Japan
}

Corresponding author: N Sweijd (nsweijd@access.ac.za)

\begin{abstract}
Concern and general awareness about the impacts of climate change in all sectors of the social-ecological-economic system is growing as a result of improved climate science products and information, as well as increased media coverage of the apparent manifestations of the phenomenon in our society. However, scales of climate variability and change, in space and time, are often confused and so attribution of impacts on various sectors, including the health sector, can be misunderstood and misrepresented. In this review, we assess the mechanistic links between climate and infectious diseases in particular, and consider how this relationship varies, and may vary according to different time scales, especially for aetiologically climate-linked diseases. While climate varies in the medium (inter-annual) time frame, this variability itself may be oscillating and/or trending on cyclical and long-term (climate change) scales because of regional and global scale climate phenomena such as the El-Niño southern oscillation coupled with global-warming drivers of climate change. As several studies have shown, quantifying and modelling these linkages and associations at appropriate time and space scales is both necessary and increasingly feasible with improved climate science products and better epidemiological data. The application of this approach is considered for South Africa, and the need for a more concerted effort in this regard is supported.
\end{abstract}

S Afr Med J 2015;105(12):1018-1023. DOI:10.7196/SAMJ.2015.v105i12.10332

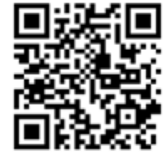

This month, the 21st Conference of Parties of the United Nations Framework Convention on Climate Change meets in Paris to attempt once again to reach an agreement on mitigation targets to reduce the now universally accepted impacts on climate change on the earth system, including the social-ecological-economic system (SEES). While the debate to date in this forum has been focused mainly on providing a sound basis for mitigation action by nation states, the focus will now need to turn to actionable information in a variety of sectors of the SEES. One of these sectors is the health sector, and specifically those diseases of which the aetiology comprises an element of climate as a driver of their incidence and epidemiology. Of particular concern are infectious diseases, vector mediated or other, which are likely to be affected by trending climate variability or climate change directly. For several decades now, as long-term climate change (as distinct from shorter-term climate variability) has become a better understood and appreciated phenomenon, climate's potential impact on health has received increasing attention (all time and space scales). While there is a broad range of potential impacts of climate and climate change on health (including heat tolerance and morbidity, for example), in this article we consider the specific mechanisms of the relationship between climate variability/change and the incidence of infectious diseases in South Africa (SA).

\section{Explaining climate terms}

It is important that three inter-related but distinct notional time and space scales of climate dynamics be differentiated in order to understand the relationship between climate and health and how it manifests. Annual seasonal periodicity manifests in typical seasonal weather patterns locally, and these can vary in duration and intensity intra- and inter-annually. For example, the frequency and intensity of heat waves, floods, storms, droughts, winds or even calm days, dryerthan-average Western Cape winters' impact on water availability in the following summer (e.g. dam levels), or last summer's properties v. this summer's properties all vary on a range of time scales. At the inter-annual to multi-year time scale $(<$ decadal), regional to hemispheric climatic configurations or climate modes (viz. El Niño and La Niña, or the El-Niño southern oscillation (ENSO)), which 
may recur in several consecutive years or occur periodically, have an impact on the properties of climate (the average weather) at a local to regional scale (often globally). These two notional time scales are commonly considered to comprise 'climate variability', whereas 'climate change' as a phenomenon considers the changes and trends in the longer-term decadal to multi-decadal climate properties (on global to local space scales) and how these are likely to trend into the future. Here, the term 'climate' is used generically to refer to all these time scales unless specified.

\section{Understanding climate in relation to health in general}

The predictability of climate variability is important if the relationship between it and infectious disease incidence is to be determined retrospectively, in order to confidently predict its impact in a changing or trending climate regime (i.e. medium-term climate variability over climate change time scales). Several reviews have been published that have identified the importance of climate variability and climate change as a factor in health and epidemiology in general. ${ }^{[1-3]}$ Epstein $^{[4]}$ reviewed several examples of how climate change and variability (and extreme weather events) have been associated with the emergence and re-emergence of infectious diseases, either directly (as a result of the biology of vector-borne diseases) or indirectly by, for example, altering the hydrological cycle. Kolstad and Johansson ${ }^{[5]}$ took a generic approach in examining the potential impact of long-term climate projections on diarrhoea in low latitudes and showed that with the most conservative estimates the incidence would increase by $8-11 \%$ in their study areas, concluding that in the face of statistical climate change uncertainty, an urgent need for more empirical research exists. In more detailed examples, the role of varying climate modes (e.g. ENSO) and their impact on seasonal and annual climate variability have been linked to variability in disease incidence. ${ }^{[6,7]} \mathrm{Zell}^{[8]}$ pointed out that the relationship between climate and health is complex in that a range of mediated drivers influence it. While seasonal and inter-annual variability can explain much of the variation in disease incidence in these reviewed studies, periodic outbreaks of disease are more likely to be due to longer-term (inter-annual to multi-decadal scale) variability via the impact of ENSO and the quasi-biennial oscillation ( $\mathrm{QBO}$ ) as examples. This is particularly demonstrable with cases involving vector-borne infectious diseases where the agent of infection is subject to environmental life cycle drivers (e.g. malaria). Zell ${ }^{[8]}$ cautioned that these climatic influences act in concert with a range of other natural (or ecologically impacted climate-mediated effects) and human-mediated drivers of disease, and unless concerted efforts are made to examine the contribution of these various drivers, they will be impossible to discern. The impacts of El Niño are most obviously detectable on the west coast of the Pacific, and the work done by Checkley et al..$^{[9]}$ is an excellent example of how these inter-annual phenomena can impact on health. In this case (for a particular ENSO event) they not only showed a departure from 'normal' (or long-term average) seasonality of disease incidence patterns (associated with a departure from normal seasonal temperature variability), but were able to calculate the risk of increased disease incidence with each degree of El Niñoattributed winter warming, and a monetary cost associated with these effects.

\section{Climate variability and change in southern Africa}

The fact that more concerted and recent climate science outputs in SA have revealed that trends and features of climate variability are indeed measurable has created an important opportunity to interrogate the relationship between climate and disease in southern Africa, regionally and in respective subregions. If the contribution of climate variability to the variability in infectious disease incidence can be determined on a range of time and space scales, the impact of these climate trends (both observed and projected) on health can then be more accurately estimated. This would provide a basis for a range of health system interventions and planning (possibly in synergy with those for other non-climate drivers of disease incidence) at appropriate time and space scales.

At the climate variability time scale, while the effects of ENSO are less consistent in southern Africa, Pohl et al..$^{[10]}$ Reason et al. ${ }^{[1]]}$ and Landman and Beraki ${ }^{[12]}$ show that seasonal scale climate variability over southern Africa is variably predictable and in some cases (particularly in the north-east) strongly associated with the ENSO state. Lazenby et al. ${ }^{[13]}$ have considered the prediction skill of temperature variability at the seasonal time scale for various time frames (in months) and various lead times and show the utility (and current limitations) of these predictabilities in respect of health applications. This is the approach adopted in a study currently underway in the Mopani district of Limpopo Province which aims to link climate and disease models for malaria, diarrhoea and pneumonia (Fig. 1). Given that some correlation between temperature and malaria incidence is apparent in the region as a whole ${ }^{[14]}$ the importance of high-resolution and long-term interrogation of these relationships is essential. The Infectious Diseases Early Warning System project (iDEWS) is collaboration among several SA and Japanese partners, which depends on this seasonal predictability skill to improve infectious disease management (Fig. 2).

In the Western Cape, this is a more complex challenge. Phillipon et al. ${ }^{[15]}$ showed that there are statistically demonstrable effects of ENSO impacts on Western Cape rainfall (with a focus on the winter months when rainfall is highest). They contend that both trends and inter-annual shifts (variability) in the position and intensity of frontal systems that bring rainfall to the Western Cape are mediated by ENSO events. Some of these effects are also discernible at high resolution, suggesting that relatively local climate impacts are long range (in space and time) drivers of climate variability. ${ }^{[16]}$ These studies suggest that rainfall and temperature variability (and conversely the Western Cape summer drought) do possess an element of predictability, and at a range of lead times.

When the data set presented in Figs 3 and 4 is considered, both intra- and annual variability in the incidence of diarrhoea is apparent in the Western Cape. Table 1 shows an as yet unexplained shift in peak month from January to March over a four-decade period. This leads to the compelling question as to whether the manifestation of variability in this dataset is attributable to climate variability at several time (and space) scales, and if so, whether the relationship can be mathematically determined for application to disease management at short-, medium- and long-term (climate change) time scales.

On the climate change time scale, analysis on observed 50-year climate data trends in southern Africa has shown important directional changes with increased annual average temperatures, as well as increased temperature maxima and minima both seasonally and on average. ${ }^{[17]}$ Extreme high temperature events have increased significantly in frequency. While the trends in rainfall are less significant and weaker, they do show a tendency towards a drier climate with more intense rainfall events ${ }^{[17]}$ in the south and west of the region, with likely impacts on daily to seasonal rainfall patterns. ${ }^{[18]}$ Model projections of various climate scenarios are variable, possess a high degree of uncertainty and are in some cases contradictory. Projections for temperature changes are more robust than those for 


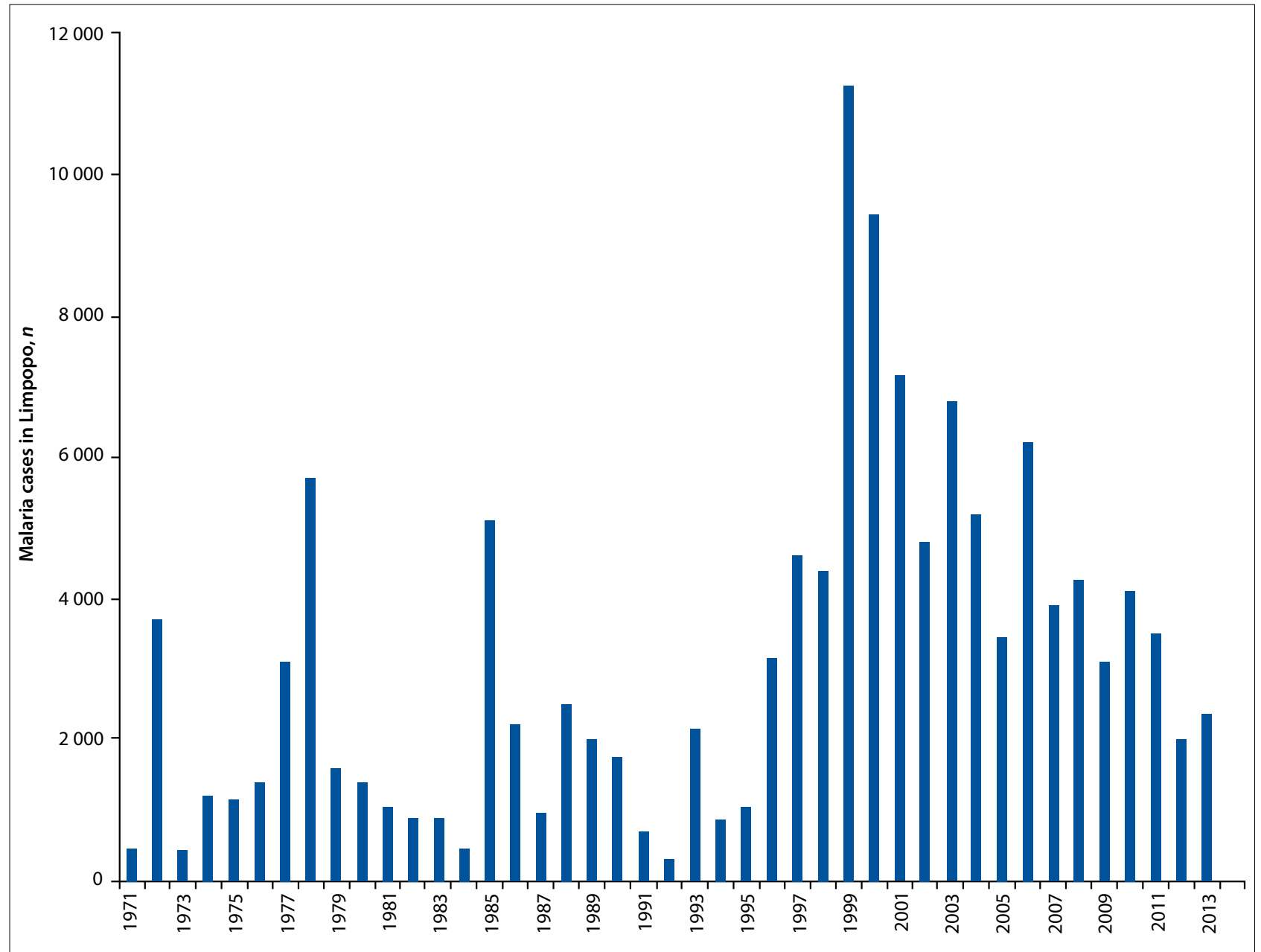

Fig. 1. The incidence of recorded malaria cases in Limpopo Province from 1976 to 2013, while restricted to the summer season, varies inter-annually in an unexplained manner. While increased incidence since the mid-1990s may be artefactual or climate driven, the opportunity to investigate the contribution climate drivers now exists. (After Kruger, personal communication.)

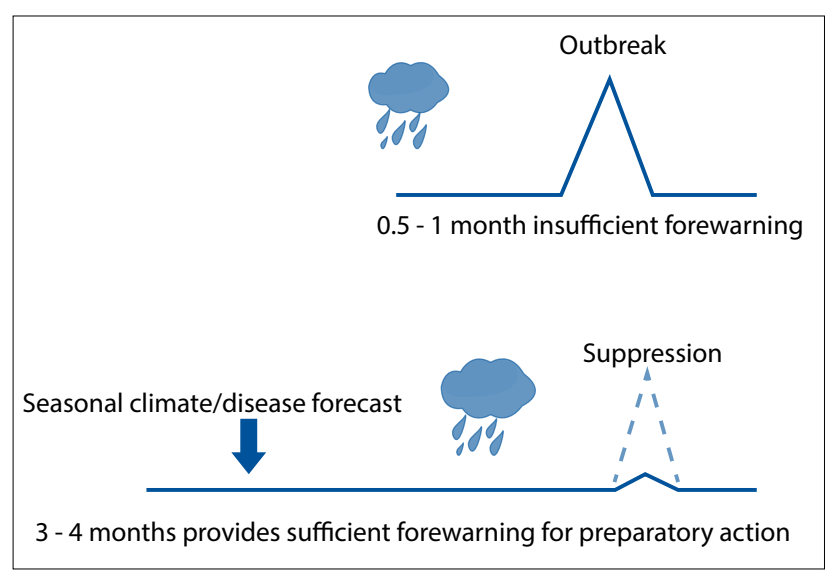

Fig. 2. Conceptual representation of iDEWS goals. Providing a seasonal scale climate-disease outlook which quantifies the likelihood of incidence increases or outbreaks will provide the opportunity for improved management interventions aimed at suppressing the impact.

precipitation, but the accepted conclusion is that, under a high $\mathrm{CO}_{2}$ emissions scenario, significant temperature increases (somewhat ameliorated in coastal areas) and a drier climate in the west of the country are predicted. ${ }^{[17]}$ While the impact on the human health sector is considered in the long-term adaptation scenarios (LTAS), ${ }^{[17]}$ the need for further concerted investigation is a common cause. ${ }^{[19]}$

\section{What do we know about climate change and health in SA?}

There are very few empirical studies on the relationship between climate and health conducted in SA in general; however, the topic has received attention with a range of publications in the grey literature and course resolution review information. One of the reasons for this is a lack of comparable and consistent long-term data (a global constraint on both climate and disease incidence). As stated above, there is certainly adequate awareness of the potential of climate change impacts on health in particular, as iterated in several articles and review papers. ${ }^{[19-22]}$ These publications generally recognise the theoretical direct and indirect impacts of climate change on health generally and infectious diseases specifically (but not exclusively), and suggest various adaptation measures and research challenges that need to be met. The literature is therefore quite clear (both internationally and locally) that the interdisciplinary approach to this topic is a vital next step. One international study, the ISOTHURM study, which considered the relationship between heat and cold thresholds associated with mortality, included Cape Town as one of the several international cities. ${ }^{[23]}$ This study was mostly descriptive and attempted to draw global conclusions about climate drivers 
of mortality. As it happened, Cape Town fell out as more of an exception in many respects, as could have been expected given the moderate Mediterranean climate, with a peak in mortality in the winter months due to respiratory illness (associated with cold and wet winters).

Mendelsohn and Dawson ${ }^{[2]}$ analysed a range of oceanographic parameters in association with a cholera outbreak in KwaZuluNatal in $2001-2002$ and found a strong association of sea surface temperature, sea surface height and precipitation with disease incidence. While the coastal transmission hypothesis is specific to cholera, this study does nevertheless provide an example of how local scale environmental variability can be used toward developing an early-warning system for disease. Thompson et al. ${ }^{[25]}$ studied the relationship between disease and mortality and climate/weather data in Limpopo. They used two decades' data for

Table 1. Diarrhoea cases admitted during peak season to Red Cross War Memorial Children's Hospital's Rehydration Unit, Cape Town, per decade showing a shift in the peak months from January to March over this period ${ }^{*}$

\begin{tabular}{llllll}
\hline & \multicolumn{4}{c}{$\begin{array}{c}\text { Frequency of annual peak in summer } \\
\text { diarrhoea cases by month (\%) }\end{array}$} \\
\cline { 2 - 5 } Decade & January & February & March & April & Total \\
\hline $1976-1985$ & 40 & 20 & 30 & 10 & 100 \\
$1986-1995$ & 40 & 20 & 40 & 0 & 100 \\
$1996-2005$ & 0 & 20 & 80 & 0 & 100 \\
$2006-2015$ & 0 & 0 & 100 & 0 & 100 \\
"After A Westwood, personal communication. & & & & &
\end{tabular}

their analysis, which is insufficient to draw conclusions in respect of climate change (their claim), but is more pertinent at the climate variability scale. Their conclusion was that around $40 \%$ of incidence of disease was attributable to climatic factors. Komen $e t$ al. ${ }^{[14]}$ showed that, statistically, temperature was a greater influence on malaria variability than rainfall for the period 1998 - 2007, and that long-term relationships between climate variables and malaria are indicated.

Despite the lack of precise information, the National and Western Cape governments have developed some policy frameworks to address climate change scale impacts and adaptation planning specific for climatehealth impacts (e.g. LTAS $^{[17]}$ ). Each of these does address the need for an integrated approach to climate and health management (among many other impact areas).

Lazenby et al. ${ }^{[13]}$ considered the application of improved seasonal climate forecasting techniques in predicting extreme seasonal temperature in the northern parts of SA, and the direct impact on health. They conclude that the skill of these forecasts is good

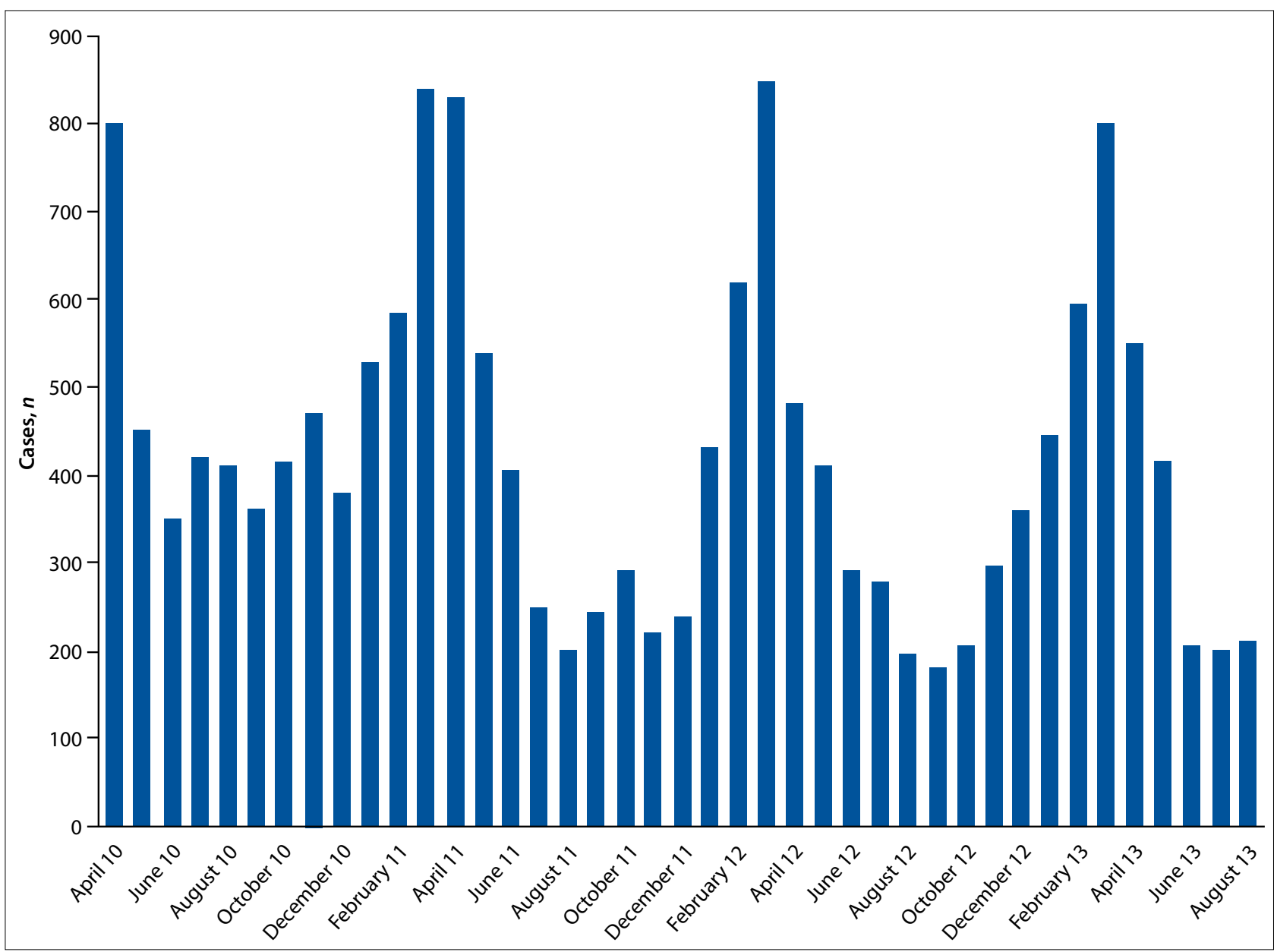

Fig. 3. Diarrhoea cases (with dehydration) for 2010 - 2013 in the Western Cape public health system demonstrate the strongly seasonal incidence of paediatric diarrhoea (confirming climate as a key driver). (After A Westwood, personal communication.) 


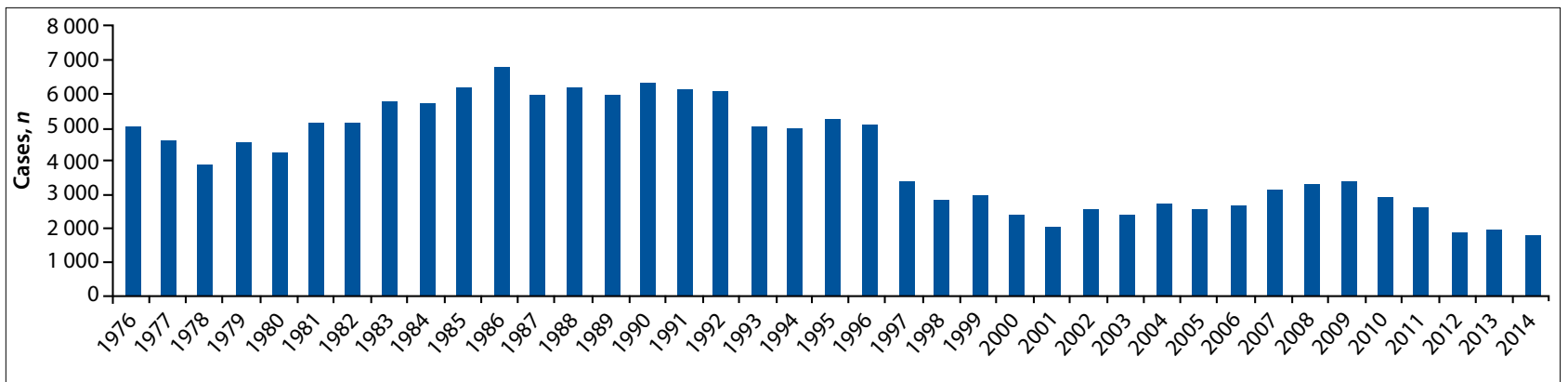

Fig. 4. Annual average variability in diarrhoea incidence in the Western Cape, from 1976 to 2014. Although policy changes have artefactually resulted in decreased admissions since 1997, a long-term oscillation in incidence is apparent. (After A Westwood, personal communication.)

enough to develop a heat-health warning system for the country. On a larger time and space scale, Garland et al. ${ }^{[26]}$ have shown how 'extreme apparent temperature' days are likely to increase over the continent under likely climate change mitigation scenarios, and they consider the implications for human health and public health management in various African hotspots.

\section{Regional and international perspective} The literature has many international (including African) examples of studies examining the relationship between climate and disease at a range of time and space scales. Some of these studies are useful indicators of the increasing likely success of these approaches using more sophisticated and/or tailored climate indices and more modern analytical techniques and mathematical models. Cholera has had the most attention in this respect, with Pascual et al. ${ }^{[27]}$ considering the geographical distribution of seasonality and inter-annual variability in the association of cholera and climate in India. Constantin de Magny et al. ${ }^{[28]}$ conducted a study examining the association of specific global and regional climate variability indices in relation to variability in cholera incidence in Ghana. Their results again confirmed a strong statistical relationship between the time series of climate and disease periodicity and incidence and show how a range of time scales (from seasons to years) can be accounted for by respectively associated global and local climate indices. In a very useful contribution, Bandyopadhyaya et al. ${ }^{[29]}$ used climatically defined areas to match with the prevalence of diarrhoea in these (sometimes transboundary) climate zones in sub-Saharan Africa (not including SA). They then tested a range of variables including climatic and non-climatic variables using a series of statistical tests based on a linear model that accounted for the chosen variables. Their results show that low rainfall in the dry season (i.e. anomalously dry) increases the prevalence of diarrhoea and that an increase in monthly average maximum temperature also drives up incidence, while an increase in monthly minimum temperature reduces incidence. This is a very good demonstration of the statistical predictability of the impact of climate on diarrhoeal illness. A recent study from Kenya ${ }^{[30]}$ has shown how important intra-annual and inter-annual seasonal climate signals are in understanding malaria incidence dynamics on a fine temporal and spatial scale. Interestingly, results are also emerging that show how contagious diseases may also have an association with climate variability. Oluwole ${ }^{[31]}$ deals with an example of meningitis epidemics in central and east Africa and their positive association with medium-term climate in the form of ENSO and other climate modes. He concludes that ENSO forecasts should be incorporated into disease management practices.

Several fairly detailed studies in other parts of the world have attempted to examine the association with climate variability (and climate change) and diarrhoeal disease incidence, with varied success. Bhandari et al. ${ }^{[32]}$ failed to show conclusive associations between their selected medium-term climatic variables and disease incidence, despite trending data in both sets of parameters. This is likely to be the result of methodological issues which in this case relied on simple regressions (as opposed to more complex nonlinear model approaches). Other common downfalls of the analyses performed include inappropriate climatic indices, poorly associated time frames for the data sets (in terms of periodicity of the climate signals and short time frames of the data sets), and also respective resolution of the data sets. Chou et al. ${ }^{[33]}$ took a more comprehensive view in examining this approach in Taiwan and applied a climate variation-guided Poisson regression model to predict the dynamics of diarrhoea-associated morbidity. They were able to show that increased maximum temperature was the primary driver of morbidity in children, with days of high relative humidity and extreme rainfall also contributing in other age groups. This information was useful in developing an early-warning system for the region. Many studies use climate variability associations to make assumptions about long-term climate change scale impacts. ${ }^{[32,34]}$ While this is risky because of the uncertainty around climate change predictions, the basic assumption is that disease incidence will trend in relation to trending climate variables, based on their current relationship, despite a range of potentially unanticipated changes and dynamics in the disease aetiology and climate properties and their relationship. In this respect, the contribution of Moors et al. ${ }^{[35]}$ is important as the authors have combined a conceptual model (which addresses the mechanisms of the relationship between climate and health), using an assessment of a range of other studies' empirical (short-term) results, and long-term climate projections to make predictions on the impact of climate change on diarrhoeal diseases in the Ganges basin in India. This approach combines different time scales and different causal pathways and is a novel and innovative approach.

Another factor to consider is which agent (i.e. specific cause of infection) is under consideration. Most studies do not differentiate the agent of disease, mostly because incidence data reflect only the symptoms at presentation and do not include any laboratory follow-up. The importance of this is illustrated by D'Souza et al. ${ }^{\left[{ }^{[6]}\right]}$ who considered the climatic impact on rotavirus infections in children in Australia. They surmise that agent-specific characteristics of transmission explain why an increase in incidence of rotaviral diarrhoeal disease coincides with a drop in temperature (seasonally) when children are confined indoors and person-to-person transmission is facilitated.

Finally, the statistical and modelling approach to the time series analysis and the association/relationship between climate and disease varies significantly among studies in the literature. This is obviously an important and specialised element of the research which must be carefully considered with the assistance of several methodological publications that address this aspect. ${ }^{[37,38]}$ 


\section{Concluding remarks}

As more sophisticated and fine-scale climate data and climate modelling outputs emerge, so too do the opportunities to apply this technology to the study of the incidence and epidemiology of (even partially) climate-driven diseases. Climate signals occur at several scales in space and time and the appropriately scaled information should be used to investigate the covariance of climate signals and disease incidence. This means that cognisance of the responses of disease to short-, medium- and long-term climate signals must be considered. The study of the relationship between climate and disease (infectious and other) is also dependent on good epidemiological data that can be aggregated and disaggregated at the right time and space scales to match with the occurrence of those of the climate signals. It is clear, however, that if effective management of climatedriven diseases is to be effected, especially in the light of trending and changing climate signals, and where this component of the aetiology of these diseases is set to become more intense with time, urgent and concerted research should be directed at quantifying this relationship for the past and present, in order to increase confidence of predicting the impact of these trends and changes in the future.

\section{References}

1. Patz JA, Grabow ML, Limaye VS. When it rains, it pours: Future climate extremes and health. Annals of Global Health 2014;80(4):332-344. [http://dx.doi.org/10.1016/j.aogh.2014.09.007]

2. Franchini M, Mannucci PM. Impact on human health of climate changes. Eur J Intern Med 2015;26(1):1-5. [http://dx.doi.org/10.1016/j.ejim.2014.12.008]

3. Watts N, Adger WN, Agnolucci P, et al. Health and climate change: Policy responses to protect public health: The Lancet Commissions. Lancet 2015;6736(15):60854-60856. [http://dx.doi.org/10.1016/ S0140-6736(15)60854-6].

4. Epstein PR. Climate change and emerging infectious diseases. Microbes Infect 2001;3(9):747-754.

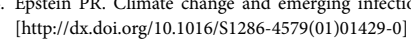

5. Kolstad EW, Johansson KA. Uncertainties associated with quantifying climate change impacts on human health: A case study for diarrhoea. Environ Health Perspect 2011;119(3):299-305. [http:// dx.doi.org/10.1289/ehp.1002060]

6. Lipp EK, Huq A, Colwell RR. Effects of global climate on infectious disease: The cholera model. Clini Microbiol Rev 2002;15(4):757-770. [http://dx.doi.org/10.1128/CMR.15.4.757-770.2002]

7. Kovats RS, Bouma MJ, Hajat S, Worrall E, Haines A. El Niño and health. Lancet 2003;362(9394):14811489. [http://dx.doi.org/10.1016/S0140-6736(03)14695-8]

8. Zell R. Global climate change and the emergence/re-emergence of infectious diseases. Int J Med Microbiol 2004;293(37):16-26. [http://dx.doi.org/10.1016/S1433-1128(04)80005-6]

9. Checkley W, Epstein LD, Gilman RH, et al. Effects of El Niño and ambient temperature on hospital admissions for diarrhoeal diseases in Peruvian children. Lancet 2000;355(9202):442-450. [http:// dx.doi.org/10.1016/S0140-6736(00)82010-3]

10. Pohl B, Fauchereau N, Reason CJC, Rouault M. Relationships between the Antarctic oscillation, the Madden-Julian oscillation, and ENSO, and consequences for rainfall analysis. J Clim 2010;23(2):238254. [http://dx.doi.org/10.1175/2009JCLI2443.1]

11. Reason CJC, Landman W, Tennant W. Seasonal to decadal prediction of southern African climate and its links with variability of the Atlantic Ocean. Bulletin of the American Meteorological Society 2006;87(7):941-955. http://adsabs.harvard.edu/abs/2006BAMS...87..941R (accessed 9 November 2015).

12. Landman WA, Beraki A. Multi-model forecast skill for mid-summer rainfall over southern Africa. International Journal of Climatology 2010;32(2):303-314. [http://dx.doi.org/10.1002/ joc.2273] Lazenby MJ, Landman WA, Garland RM, deWitt DG. Seasonal temperature prediction skill over southern Africa and human health. Meteorological Applications 2014;21(4):963-974. [http:// dx.doi.org/10.1002/met.1449]
13. Komen K, Olwoch J, Rautenbach H, Botai J, Adebayo A. Long-run relative importance of temperature as the main driver to malaria transmission in Limpopo Province, South Africa: A simple econometric approach. Ecohealth 2015;12(1):131-43. [http://dx.doi.org/10.1007/s10393-014-0992-1]

14. Phillipon N, Rouault M, Richard Y, Favre A. The influence of ENSO on winter rainfall in South Africa. International Journal of Climatology 2012;32(15):2333-2347. [http://dx.doi.org/10.1002/joc.3403]

15. Dufois F, Rouault M. Sea surface temperature in False Bay (South Africa): Towards a better Dufois $\mathrm{F}$, Rouault $\mathrm{M}$. Sea surface temperature in False Bay (South Africa): Towards a better
understanding of its seasonal and inter-annual variability. Cont Shelf Res 2012;4:24-35. [http://dx.doi. understanding of its seasonal and

16. LTAS. Long-Term Adaptation Scenarios Flagship Research Programme (LTAS) for South Africa. Climate Trends and Scenarios for South Africa. Pretoria: Department of Environmental Affairs, 2013. https://www. environment.gov.za/sites/default/files/docs/biodiversity_BookV4.pdf (accessed 11 November 2015).

17. Fauchereau S, Trzaska S, Rouault M, Richard Y. Rainfall variability and changes in southern Africa during the 20th century in the global warming context. Natural Hazards 2003;29(2):139-154. [http:// dx.doi.org/ 10.1023/A:1023630924100

18. Ziervogel G, New M, Archer van Garderen E, et al. Climate change impacts and adaptation in South Africa. WIREs Climate Change 2014;5(5):605-620. [http://dx.doi.org/10.1002/wcc.295]

19. Myers J, Young T, Galloway M, Manyike P, Tucker T. Responding to climate change in southern Africa - the role of research. S Afr Med J 2011;101(11):820-822.

20. Myers JE, Rother H-A. Public health impact of and response to climate change in South Africa. South African Health Review 2012/13:127-138. http://www.health-e.org.za/wp-content/uploads/2013/04/ SAHR2012_13_lowres_1.pdf (accessed 9 November 2015).

21. Wright CY, Garland RM, Norval M, Vogel C. Human health impacts in a changing South African climate. S Afr Med J 2014;104(8):579-582. [http://dx.doi.org/10.7196/SAMJ.8603]

22. McMichael AJ, Wilkinson P, Kovats RS, et al. International study of temperature, heat and urban mortality: The 'ISOTHURM' project. Int J Epidemiol 2008;37(5):1121-1131. [http://dx.doi.org/10.1093/ije/dyn086]

23. Mendelsohn J, Dawson T. Climate and cholera in KwaZulu-Natal, South Africa: The role of environmental factors and implications for epidemic preparedness. Int J Hygiene Environ Health 2008;211(1-2):156-162.

24. Thompson AA, Matamale L, Shonisani DK. Impact of climate change on children's health in Limpopo province South Africa. Int J Environ Res Public Health 2012;9(3):831-854. [http://dx.doi.org/10.3390/ijerph9030831]

25. Garland R, Matoaane M, Engelbrecht E, et al. Regional projections of extreme apparent temperature days in Africa and the related potential risk to human health. Int J Environ Res Public Health 2015;12(10):12577-12604. [http://dx.doi.org/10.3390/ijerph121012577]

26. Pascual M, Bouma MJ, Dobson AP. Cholera and climate: revisiting the quantitative evidence. Microbes Infect 2002;4(2):237-245

27. Constantin de Magny G, Cazelles B, Guegan J-F. Cholera threat to humans in Ghana is influenced by both global and regional climatic variability. EcoHealth 2007;3(4):223-231. http://link.springer.com/ article/10.1007/s10393-006-0061-5 (accessed 9 November 2015).

28. Bandyopadhyaya $S$, Kanjib $S$, Wanga $L$. The impact of rainfall and temperature variation on diarrheal prevalence in sub-Saharan Africa. Appl Geogr 2012;33(1):63-72. [http://dx.doi.org/10.1016/j.apgeog.2011.07.017]

29. Midekisa A, Beyene B, Mihretie A, Bayabil E, Wimberly MC. Seasonal associations of climatic drivers and malaria in the highlands of Ethiopia. Parasit Vectors 2015;8(1):339-350. [http://dx.doi. org/10.1186/s13071-015-0954-7]

30. Oluwole OS. Climate regimes, El Niño-Southern Oscillation, and meningococcal meningitis epidemics. Frontiers in Public Health 2015;30(3):187. [http://do.doi.org/10.3389/fpubh.2015.00187]

31. Bhandari GP, Gurung S, Dhimal M, Bhusal CL. Climate change and occurrence of diarrheal diseases: Evolving facts from Nepal. Journal of the Nepal Health Research Council 2012;10(22):181-186.

32. Chou W-C, Wu J-L, Wang Y-C, Huang H, Sung F-C, Chuang C-Y. Modeling the impact of climate variability on diarrhoea-associated diseases in Taiwan (1996-2007). Sci Total Environ 2010;409(1):4351. [http://dx.doi.org/10.1016/j.scitotenv.2010.09.001]

33. Singh RBK, Hales S, de Wet N, Raj R, Hearnden M, Weinstein P. The influence of climate variation . Singh RBK, Hales S, de Wet N, Raj R, Hearnden M, Weinstein P. The influence of climate variation
and change on diarrheal disease in the Pacific islands. Environ Health Perspect 2001;109(2):155-159.

34. Moors E, Singh T, Siderius C, Balakrishnan S, Mishra A. Climate change and waterborne diarrhoea in northern India: Impacts and adaptation strategies. Sci Total Environ 2013;468/469(S1):139-151. [http://dx.doi.org/10.1016/j.scitotenv.2013.07.021]

35. D'Souza RM, Hall G, Becker NG. Climatic factors associated with hospitalizations for rotaviru diarrhoea in children under 5 years of age. Epidemiol Infect 2008;136(1):56-64.

36. Pourabbas E, d'Onofrio A, Rafanelli M. A method to estimate the incidence of communicable disease under seasonal fluctuations with application to cholera. Appl Math Comput 2001;118(2-3):161-174 [http://dx.doi.org/10.1016/S0096-3003(99)00212-X]

37. Leyk S, McCormick BJJ, Nuckols JR. Effects of varying temporal scale on spatial models of mortality patterns attributed to paediatric diarrhoea. Spat Spatiotemporal Epidemiol 2011;2(2):91-101. [http:// dx.doi.org/10.1016/j.sste.2011.03.002

Accepted 5 November 2015 